\title{
TOPOLOGY OPTIMIZATION OF STRUCTURES WITH STRESS AND ADDITIVE MANUFACTURING CONSTRAINTS ${ }^{1}$
}

\author{
Grzegorz Fiuk, MirosŁaW W. MrzygŁóD \\ Opole University of Technology, Opole, Poland \\ e-mail: g.fiuk@doktorant.po.edu.pl;m.mrzyglod@po.edu.pl
}

\begin{abstract}
The paper presents a new methodology dedicated to design for additive manufacturing. Based on a hybrid algorithm of topological optimization, the method enables application of advanced constraints and validates solutions "on the fly" using virtual prototyping. Advanced constraints consider the influence of directions of additive manufacturing as well as the equivalent stress. In the optimization framework, real material properties related to three manufacturing directions were considered. The new design methodology is illustrated by benchmark tests and examples of wrist-hand orthosis topology optimization in which stress and manufacturing constraints were taken into account. As demonstrated by the conducted comparison tests with available commercial tools, the solutions obtained with the new method were characterized by lower mass and shorter computation time.
\end{abstract}

Keywords: topology optimization, manufacturing constraints, additive manufacturing

\section{Introduction}

The application of the topology optimization method for additive manufacturing (AM) is very popular nowadays. However, despite the development of topology optimization tools, there are still several unsolved problems to which one can include the use of advanced constraints and the necessity of validation of the solution.

The influence of material anisotropy to an optimization result, for the additive manufacturing process, is quite a new field of research. Among large numbers of research papers about structure design for additive manufacturing, not many are considering material properties after the AM process. Zhang et al. (2017) analyzed the role of anisotropy properties to optimization for additively manufactured bearing structures. They conclude that the role of the anisotropic material model that results from AM processes needs to be considered during the optimization process. In the paper of Park et al. (2019), the authors prepared a numerical model and tested for an additively made sport rifle support. Researchers proved that the realistic material model influenced final test results for the part. Dapogny et al. (2019) proposed a new numerical model for anisotropic material properties, which depended on the manufacturing process and building technology. Also, Jiang (2017) in his Ph.D. thesis, made a complex analysis of the influence of material anisotropy on three-dimensional optimization of additive manufacturing. In the work of Mirzendehdel et al. (2018), researchers presented strength-based topology optimization with anisotropic materials for additive manufacturing, which included new failure criteria, like Tsai-Wu. Based on that state-of-the-art research, authors concluded that there was a need to compare sensitivity of different types of optimization methods for additive manufacturing technologies to anisotropic material properties. In his Ph.D. thesis, Hoglund (1992) presented topology optimization for the Fused Filament Fabrication method including anisotropy of a fiber-reinforced filament.

\footnotetext{
${ }^{1}$ This work is related to a paper presented at PCM-CMM 2019.
} 
Sheth et al. (2017) showed a procedure for numerical prediction of the Elastic Modulus of Fused Deposition Modeling (FDM) printed parts as a function of the raster angle. Also, AM design was taken by Mirzendehdel and Suresh (2016) were researchers considered optimization problems for direct additive process in terms of manufacturing support constrains. A similar issue was accompanied by Thore et al. (2019), who analysed overhung constraints for additive manufacturing, which was implemented in the optimization algorithm. In the research, Liu et al. (2019) considered cost constraints of additive manufacturing with a topology optimization method. They used real cost data of manufacturing, and applied it to the level-set function.

In this paper, a new design methodology with stress and additive manufacturing constraints is proposed. The effectiveness of the original method is compared with commercial optimization tools demonstrated by using the Lewiński-Rozvany benchmark problem (Lewiński and Rozvany, 2008) and a hand orthosis design example.

\section{Topology optimization with additive manufacturing constraints}

In the investigation, two types of optimization algorithms were taken - density-based optimization based on Solid Isotropic Material with Penalization (SIMP) (Zhou and Rozvany, 1991), and a hybrid algorithm based on evolutionary topology optimization with simulated annealing method - Constant Surface Criterium Algorithm (CSSA) (Mrzygłód, 2012). Both methods were used with ANSYS software, were SIMP optimization was already implemented tool, and CSSA was written in APDL code and launched in batch mode.

The optimization problem can be presented as follows

$$
\min _{\rho} f(\boldsymbol{\rho})
$$

The constraints are

$$
g_{j}(x) \leqslant \overline{g_{j}} \quad j=[1,2, \ldots, M]
$$

where: $\mathbf{x}=\left[x_{1}, x_{2}, \ldots, x_{N}\right]-$ is a vector of finite elements, $\boldsymbol{\rho}=\left[\rho_{1}, \rho_{2}, \ldots, \rho_{N}\right]$ - is a vector of design variables (pseudo-density of finite elements) defined as $\rho_{i}=E_{\min }$ or $E_{0}, E_{\min }$ and $E_{0}$ are, respectively, real and minimum Young's modulus of the material of the structure, $g_{j}(\mathbf{x})$ - are constraint criterion parameters, $\overline{g_{j}}$ - are the upper bounds of the constraints (condition parameters).

The CSSA algorithm can be treated as a continuation of the development of ESO/BESO methods (Xie and Steven, 1993; Querin et al., 1998). The algorithm generates a solution through iterative elimination of elements with a low value of the constraint criterion function $g_{j}(\mathbf{x})$ (see Fig. 1a). The elimination process of low-stress finite elements is controlled by a fixed percentage parameter of subtracted volumes $\Delta F$. To obtain a constant value of $\Delta F$ during each iteration, the constraint criterion value of $g_{\min }(\Delta F)$ is dynamically determined. The topological optimization procedure can give an early non-optimal solution when it stops at a point with a high value of the constraint. This means that the optimization process has achieved a local minimum. To counteract the occurrence of this effect in the CCSA algorithm, a layered growth process was introduced. In the case when the limit value of the constraint criterion is achieved, instead of removing the volume, finite element values $\Delta F$ are added to the entire surface of the structure. The volume increase procedure is continued until the criterion $g_{j}(\mathbf{x})$ returns to the acceptable range. The end of the algorithm operation controls the arbitrarily set parameter LMX of the maximum number of iterations. This procedure is analogous to the simulated annealing (Kirkpatrick et al., 1983). 
(a)

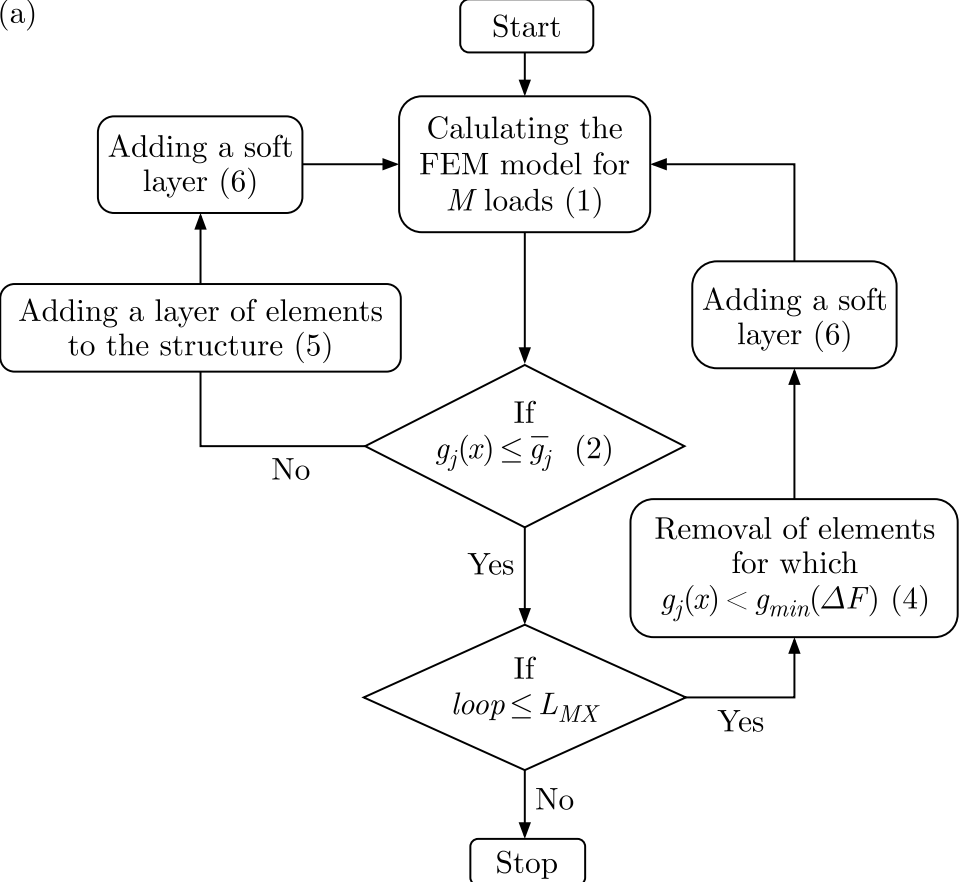

(b)

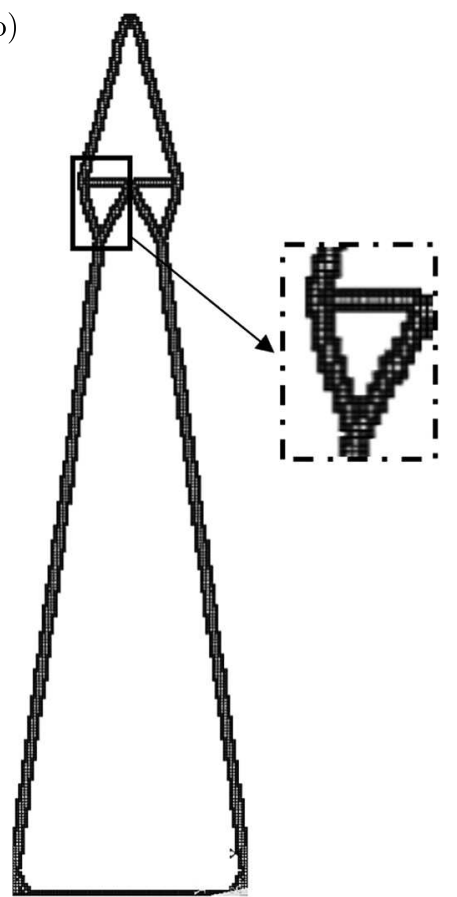

Fig. 1. Constant Criterion Surface Algorithm of topology optimization (a) and exemplary structure surrounded by the soft layer (darker colour) (b)

In the algorithm, a new procedure of surrounding solutions through the layer of finite elements with the minimum value of the Young modulus $E_{\text {min }}$ has been added. The thus created "soft layer" increases the stiffness of slender elements and improves the stability of the algorithm.

The CCSA algorithm for the problem of minimizing volume (2.1) and one constraint consists of the following steps (Fig. 1):

1. START: The procedure starts the optimization and moves to step (1).

2. Step (1): For the current FE model, $M$ cases of loads are calculated and written in the form of a vector of $h$ value of the constraint parameter $g_{j}(\mathbf{x})$ for all $N$ finite elements.

3. Step (2): Checking the maximum value of the constraint criterion $g_{j}(\mathbf{x})$ in $N$ finite elements. If it is higher than the assumed boundary value $\left(g_{j}(\mathbf{x})>\overline{g_{j}}\right)$, the procedure moves on to step (5). However, if the values $g_{j}(\mathbf{x})$ do not exceed $\overline{g_{j}}\left(g_{j}(\mathbf{x}) \leqslant \overline{g_{j}}\right)$, the procedure moves on to step (3).

4. Step (5): Adding a layer of finite elements $\left(\rho_{i}=E_{0}\right)$ to the entire surface of the current structure $\Omega_{b}$. The procedure moves to step (6).

5. Step (6): Adding a soft layer of finite elements $\left(\rho_{i}=E_{\min }\right)$ to the entire surface of the current structure $\Omega_{b}$ (see Fig. 1b). The procedure moves to step (1).

6. Step (3): Checking the number of the loop iteration. If the number of iterations does not exceed the assumed maximum value loop $\leqslant L_{M X}$, the procedure moves to step (4). In the opposite case (loop $\left.>L_{M X}\right)$, the procedure moves to STOP.

7. Step (4): Removing from the structure the group of finite elements $\Omega_{r}$ for which $g_{j}(\mathbf{x})<g_{\min }(\Delta F)$ (for the group of elements $\Omega_{r}$ the operation of subtracting by $\rho_{i}=E_{0}$ is performed). The boundary-value of the condition parameter $g_{\min }(\Delta F)$ is dynamically determined at this level, making it possible to remove the constant percentage volume value $\Delta F$ (usually $\Delta F=1 \%$ of the volume of the structural space). The remaining group of result elements creates the current solution $\left(\Omega_{b(\text { loop })}=\Omega_{b(\text { loop }-1)}-\Omega_{r}\right)$ characterized by a constant value of the constraint parameter (e.g., reduced stress) on the structure surface. The procedure moves to step (6).

8. STOP: The procedure stops the optimization. 
The solutions obtained using the CCSA algorithm are characterized by a lightweight and compact structure while meeting all accepted restrictions without the need for validation. These solutions are directly suitable for manufacturing with additive technology.

\section{Benchmark tests}

As a benchmark test, the Lewiński-Rozvany beam problem (Lewiński and Rozvany, 2008) was selected. This benchmark is based on Michel's structure (Michell, 1904). It is a rectangular beam with a square hole near one of its shorter sides. On the opposite side, there is a force applied. In this experiment, the model was oriented along three main axes $X, Y$ and $Z$. Dimensions and boundary conditions are shown in Figs. 2 and 3, and the mesh for both methods is presented in Table 2 .

In the modelling of additively made materials ULTEM 9085 were used, where material properties were tested by El-Gizawy et al. (2011) and are presented in Table 1. The data was implemented in Ansys Workbench using Engineering Data tools, for the SIMP method, and APDL material commands (MPDATA, E-direction, $P R$-direction, $G$-direction).

Table 1. Mesh parameters of the beam

\begin{tabular}{|l|c|}
\hline Method & Hex Dominant \\
\hline Size & $2 \mathrm{~mm}$ \\
\hline No. elements & 25228 \\
\hline
\end{tabular}

Table 2. ULTEM 9085 properties

\begin{tabular}{|c|c|c|c|c|c|c|}
\hline \multicolumn{2}{|c|}{ Modulus of elasticity } & \multicolumn{2}{|c|}{ Poisson ratio } & \multicolumn{2}{|c|}{ Shear modulus } & Density \\
\hline \hline$E_{1}$ & $2539 \mathrm{MPa}$ & $\nu_{12}$ & 0.46 & $X Y$ & $635 \mathrm{MPa}$ & \multirow{2}{*}{$1.25 \mathrm{~g} / \mathrm{cm}^{3}$} \\
\hline$E_{2}$ & $2327 \mathrm{MPa}$ & $\nu_{13}$ & 0.39 & $Y Z$ & $635 \mathrm{MPa}$ & \\
\hline$E_{3}$ & $2159 \mathrm{MPa}$ & $\nu_{23}$ & 0.40 & $X Z$ & $582 \mathrm{MPa}$ & \\
\hline
\end{tabular}

(a)

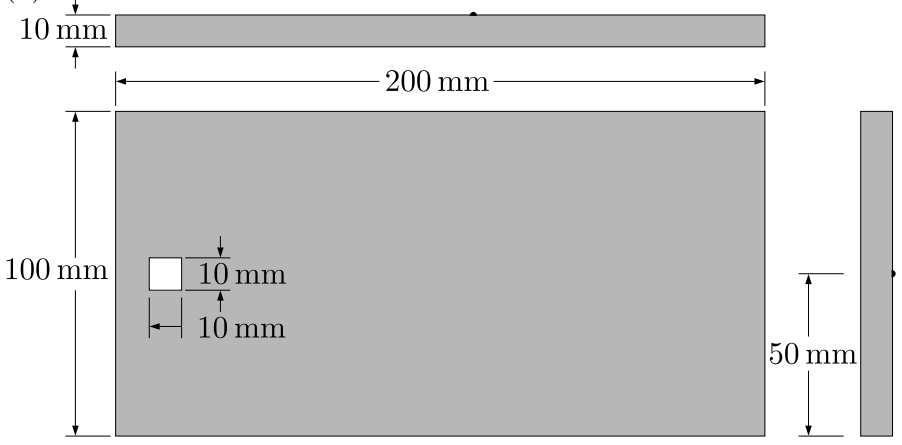

(b) A: ULTEM YX
Static structural Time: $1, \mathrm{~s}$ A Fixed support
B Force: $75, \mathrm{~N}$ (A)

Fig. 2. Dimensions (a) and boundary conditions of the beam (b)

In the beam analysis, the mass of the structure is optimized with the von Mises stress constraint $(20 \mathrm{MPa})$, with exclusion of boundaries in the optimization space.

Results of density optimization for the three main axes are shown in Fig. 3 an Table 3 showing optimization results. Next, the effect of CSSA optimization was shown with explaining the soft layer feature and stress validation with the soft layer, Figs. 5a,b. Optimization in the 
(a)

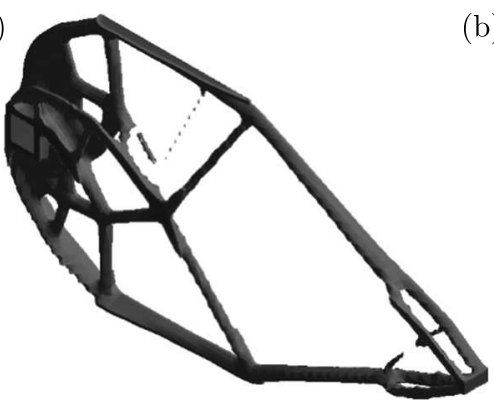

(b)

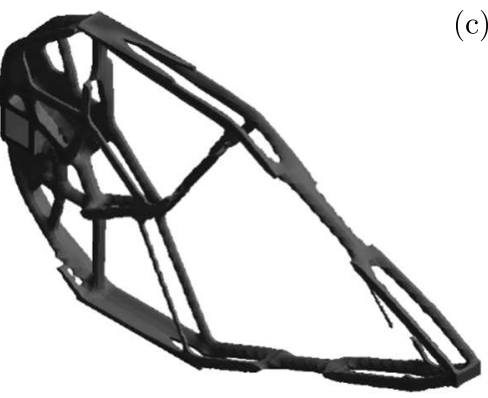

(c)

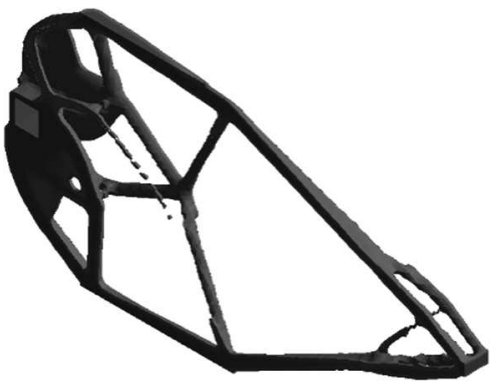

Fig. 3. Result of optimization in $Y Z$ (a), $Z X$ (b) and $Y X$ (c) direction

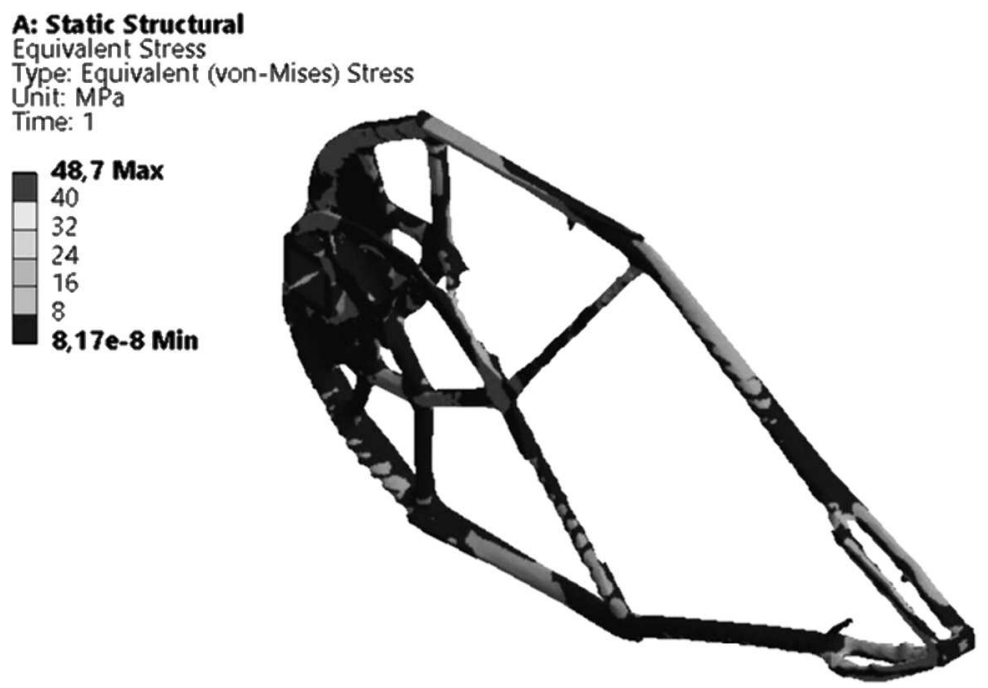

Fig. 4. Stress validation of $Y Z$ optimization

Table 3. Mass optimization density-based results

\begin{tabular}{|l|c|c|}
\hline Original mass & $248 \mathrm{~g}$ & $100 \%$ \\
\hline$Y Z$ direction & $20.42 \mathrm{~g}$ & $8.2 \%$ \\
\hline$Z X$ direction & $22.45 \mathrm{~g}$ & $9 \%$ \\
\hline$Y X$ direction & $20.73 \mathrm{~g}$ & $8.3 \%$ \\
\hline
\end{tabular}

three main axes is shown in Fig. 6, and in Fig. 7 stress validation for the $Z X$ axes for the model without stress layer. Table 4 presents the optimization results. Comparing both result series one can see that in the $Z X$ direction, density is quite bigger than in the two other directions. This can be correlated with the lowest values of modulus of elasticity and shear modulus with the highest Poisson ratio. Also, the final mass in both solutions achieves higher values for $Z X$. The final volume of structures shows differences between the methods of optimization.

The CSSA method achieves a lighter structure than the density-based method, and the difference is about $2 \%$ of the final mass. During validation of the density solution, it is needed to rebuild the model from the STL file. This step affects the proposed optimal solution because, often, engineers must change the original geometry of the solution. After rebuilding, static stimulation is performed on the same boundary conditions. The results are shown in Fig. 4. It is essential to mention that the optimized model does not require specified maximum stress, and static simulations show areas with results of 30-48.7 MPa of the Huber-Mises-Hencky equivalent stress. The CSSA validation results are in the range of stress constraints, mainly because of validation done at every iteration. 
(a)

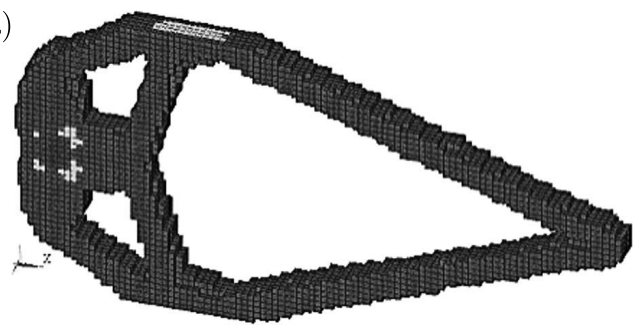

(b)

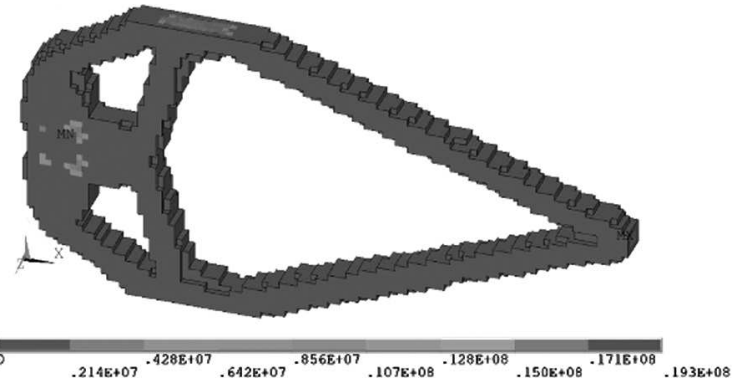

Fig. 5. Soft layer feature (darker colour) (a) and stress validation with a soft layer (b)

(a)

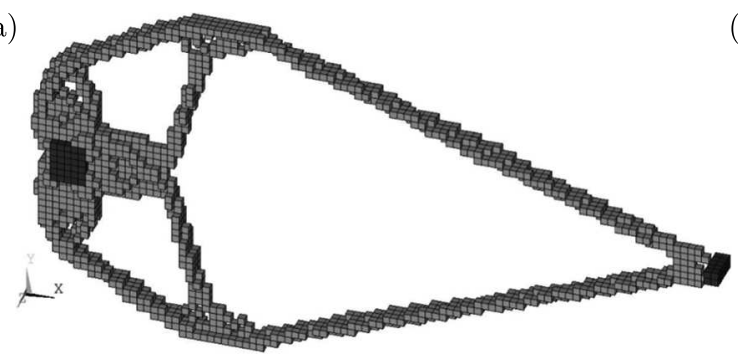

(b)

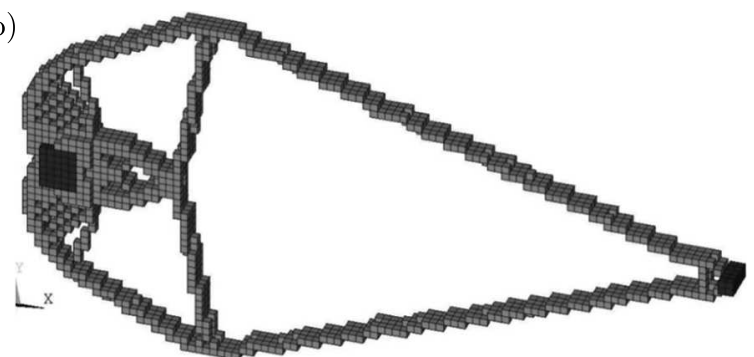

(c)

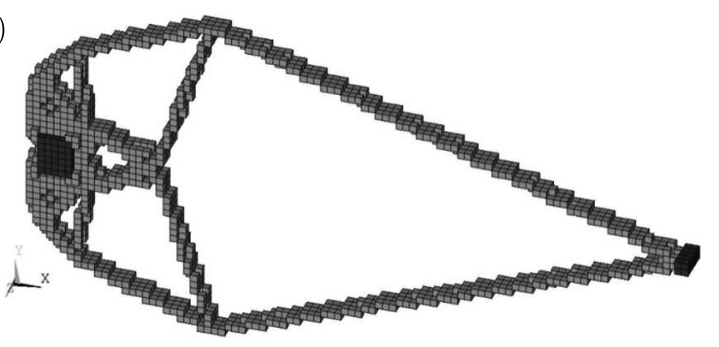

Fig. 6. Result of optimization in $Z X, Y Z$ and $Y X$ directions
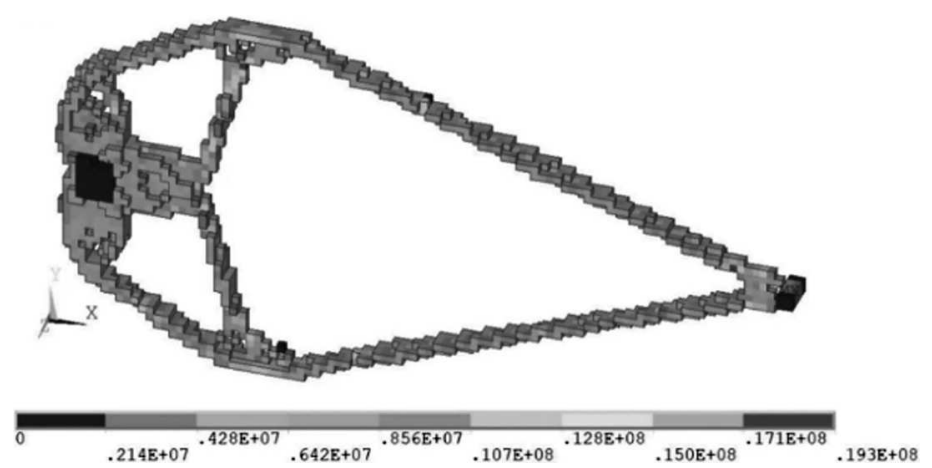

Fig. 7. Stress validation of $Z X$ optimization

Table 4. Mass optimization CSSA results

\begin{tabular}{|l|c|c|}
\hline Original mass & $248 \mathrm{~g}$ & $100 \%$ \\
\hline$Z X$ direction & $16.86 \mathrm{~g}$ & $6.7 \%$ \\
\hline$Y Z$ direction & $15.87 \mathrm{~g}$ & $6.4 \%$ \\
\hline$Y X$ direction & $14.63 \mathrm{~g}$ & $5.9 \%$ \\
\hline
\end{tabular}




\section{A design example of a wrist hand orthosis}

For the hand orthosis, the design model was prepared from the reverse-engineered scan of a patient hand (Fig. 8a). The main aim was to support the injured wrist, with an additively made orthosis, which was designed directly to patients' hand dimensions. It was designed to join in the middle section and support the wrist after the injury. The boundary condition shown in Fig. 8b is based on design joining forces, and item handling situation. For the orthosis, analysis of mesh parameters is given in Table 5. The mass of the structure is the aim of optimization with the von Mises stress constraint (40 MPa), excluding the boundaries in the optimization space.
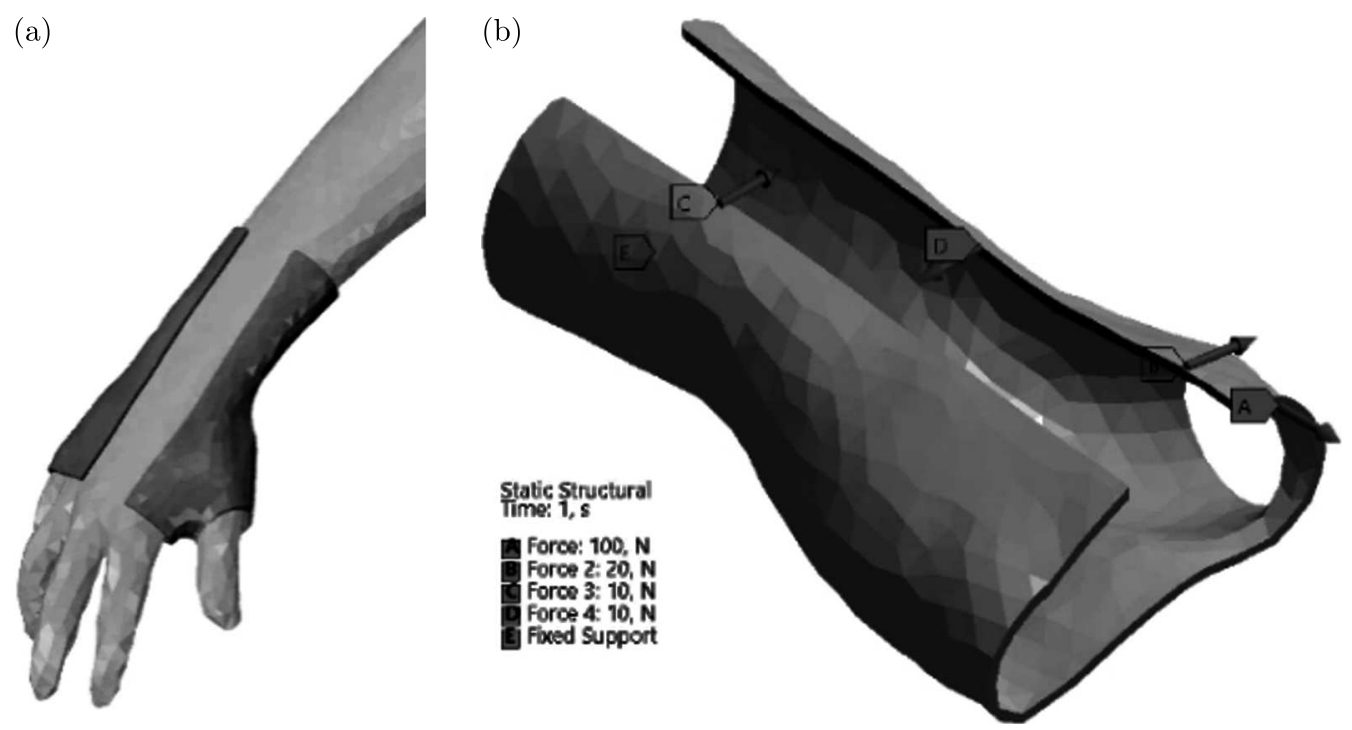

Fig. 8. Designed hand orthosis (a) and boundary conditions of FEM analysis (b)

Table 5. Mesh parameters for orthosis

\begin{tabular}{|l|c|}
\hline Method & Hex dominant \\
\hline Element size & $0.5 \mathrm{~mm}$ \\
\hline No. elements & 65698 \\
\hline
\end{tabular}
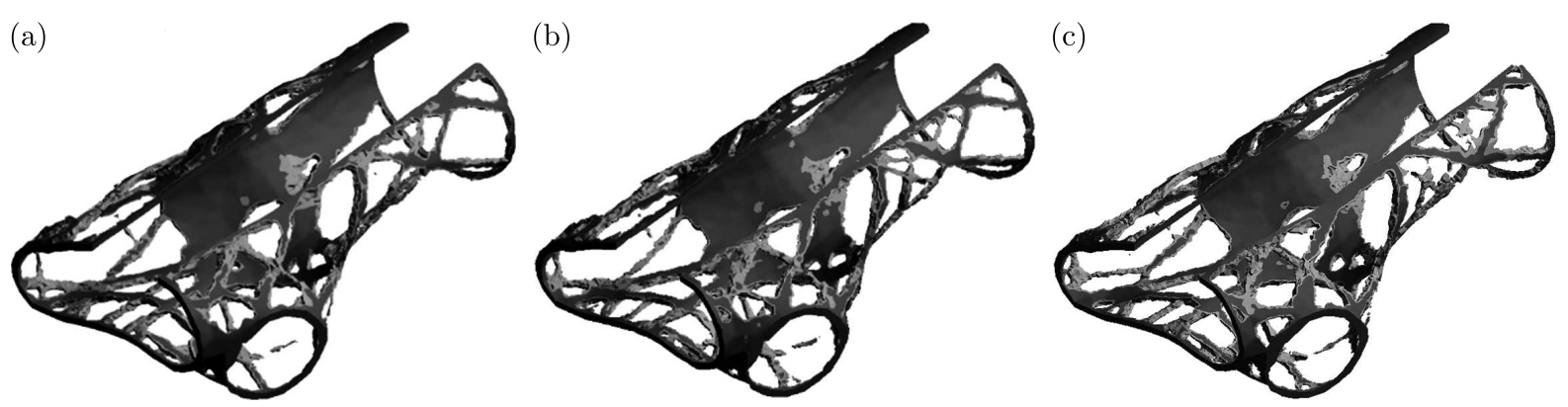

Fig. 9. Result of optimization in $Y X$ (a), $Y Z$ (b) and $Z X$ (c) AM directions

Results of density optimization in the three main axes are shown in Fig. 9 and Table 6. The best values of the optimized orthosis were achieved in the $Z X$ direction, and they were $1 \%$ worst. The validation in the $Y Z$ direction is shown in Fig. 10. As can be seen, the static analysis gove an infeasible result higher than the yield strength for ULTEM.

In Fig. 11 the effect of CSSA optimization, with explaining the soft layer feature and stress validation with the soft layer, is presented. The optimization in the three main axes is depicted in Fig. 12 and Table 7. The best results were achieved for $Y X$ and $Y Z$ directions. $Z X$ direction got a $1 \%$ less volume. Figure 13 presents stress validation for the model without the stress 
Table 6. Mass optimization density-based results

\begin{tabular}{|l|c|c|}
\hline Original mass & $39 \mathrm{~g}$ & $100 \%$ \\
\hline$Y X$ direction & $14.15 \mathrm{~g}$ & $36.3 \%$ \\
\hline$Y Z$ direction & $14.20 \mathrm{~g}$ & $36.4 \%$ \\
\hline$Z X$ direction & $13.88 \mathrm{~g}$ & $35.6 \%$ \\
\hline
\end{tabular}

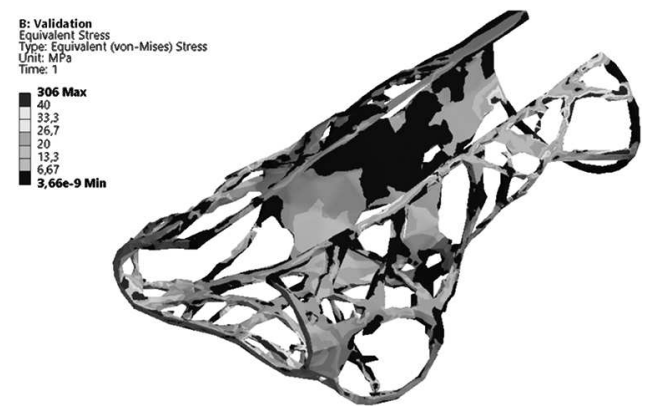

Fig. 10. Design validation of the optimization in $Y Z$ AM direction

(a)

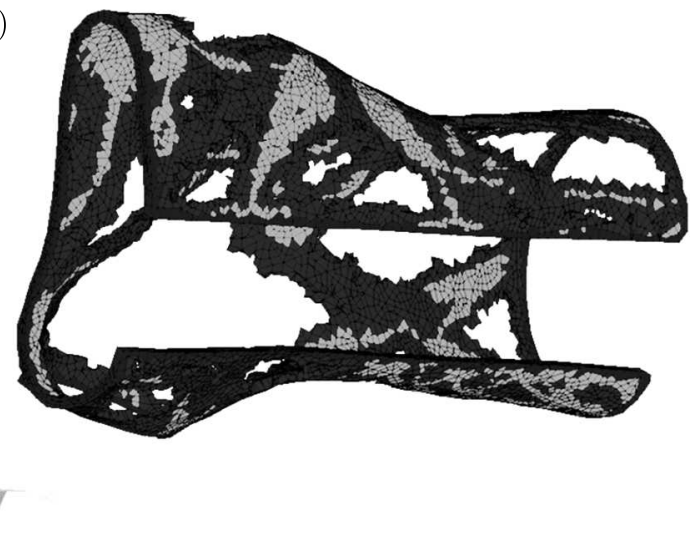

(b)

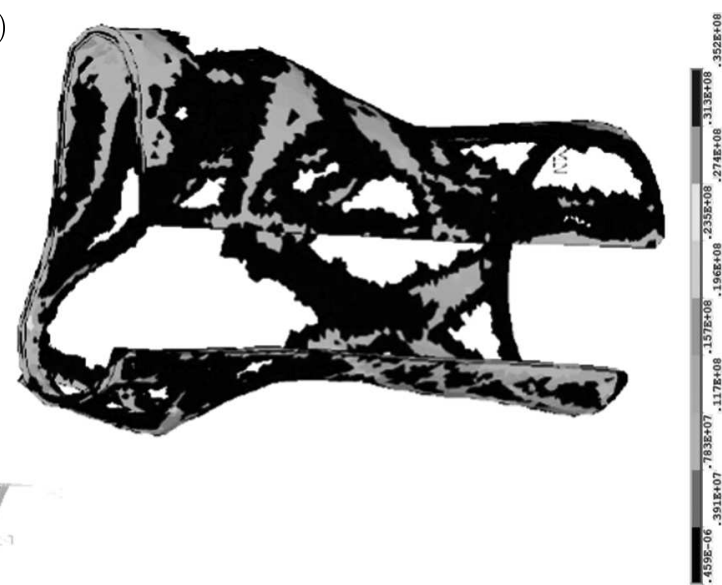

Fig. 11. Result of optimization in $Y X$ AM direction (a) and design validation with a soft layer (b) (darker colour)

(a)

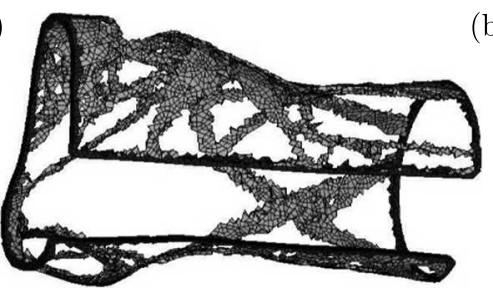

(b)

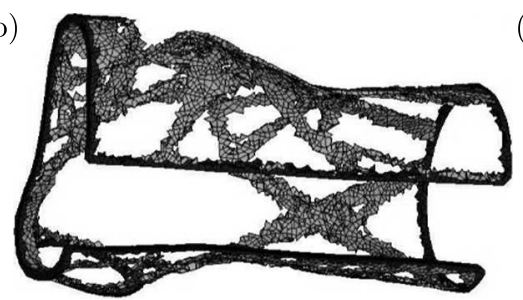

(c)

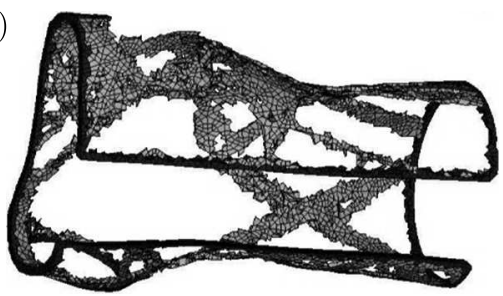

Fig. 12. Result of optimization in $Y X$ (a), $Y Z$ (b) and $Z X$ (c) AM direction

layer in the $Y X$ direction. As it is shown, the orthosis is under stress constraint due in-loop validation implemented in CSSA. It could be concluded that with complex boundary conditions, optimization results had less sensitivity to material anisotropy.

It should be noticed that the CSSA algorithm, also in the second example, allows obtaining the lower value of the objective function in the optimum solution when compared with the ANSYS/SIMP method. Moreover, the CCSA algorithm maintains stress constraints during the optimization process, whereas ANSYS/SIMP requires a difficult and complex process of validation to obtain the correct design solution. This allows us to say that the CSSA algorithm 
Table 7. Mass optimization CSSA results

\begin{tabular}{|l|c|c|}
\hline Original mass & $39 \mathrm{~g}$ & $100 \%$ \\
\hline$Y X$ direction & $8.85 \mathrm{~g}$ & $22.7 \%$ \\
\hline$Y Z$ direction & $8.85 \mathrm{~g}$ & $22.7 \%$ \\
\hline$Z X$ direction & $8.89 \mathrm{~g}$ & $22.8 \%$ \\
\hline
\end{tabular}

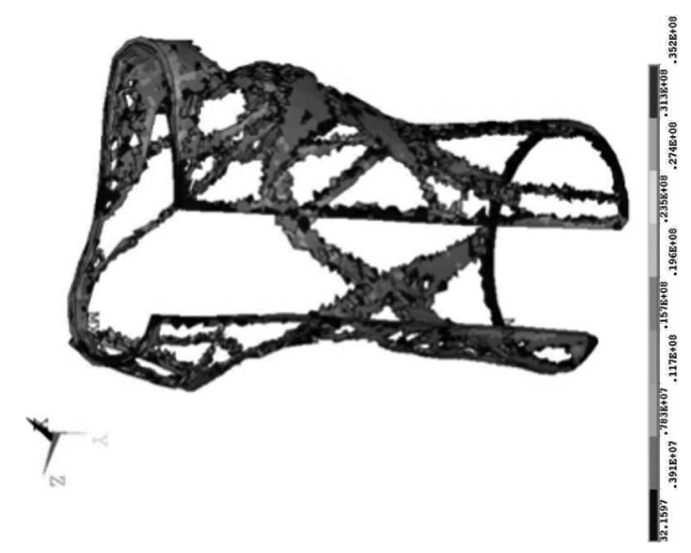

Fig. 13. Stress validation of $Y X$ optimization

could increase the efficiency by time-saving of redesigning and validating, with the opportunity of giving ready-to-print models. In these examples, the differences in favor for the CSSA method were the evidence.

\section{Conclusions}

The research aimed at finding the effective methodology of topology optimization with the application of additive manufacturing constraints. As AM constraints, real material properties related to three manufacturing directions were considered. For the AM design, the CCSA evolutionary algorithm with a soft layer procedure was used. The comparison of the effectiveness of the CCSA algorithm with ANSYS/SIMP software tool was made with the help of two numerical examples: the Lewiński-Rozvany benchmark problem, and biomechanical design of a wrist-hand orthosis. Results show high influence of the anisotropic properties of the material on the design shape, whereas the CCSA solutions revealed lower values of the objective function (volume) compared to SIMP optimization. Moreover, ANSYS framework required a time consuming process of the validation.

For the Lewiński-Rozvany benchmark with simple boundary conditions, bigger influence of material anisotropy on the layout of the design was observed. In both numerical examples, the CSSA method achieved better results of the objective function for all 3 directions of manufacturing with maintaining stress constraints. For the density-based SIMP method, the obtained solutions required an additional time-consuming validation procedure to maintain stress constraints. The presented CCSA algorithm allows obtaining "effective" solutions that meet the adopted constraints and give the possibility of direct fabrication by additive manufacturing. Moreover, the proposed methodology can be useful in design structures based on real material properties that consider the direction of manufacturing. Further investigation may concern the use of a gradient-functional material design approach as manufacturing constraints for AM.

\section{Acknowledgments}

This paper is funded by the National Science Center, Poland on the implementation of the scientific activity, which received funding under the competition: "MINIATURA I" No. 2017/01/X/ST8/00156. 


\section{References}

1. Dapogny C., Estevez R., Fuare A., Michailidis G., 2019, Shape and topology optimization considering anisotropic features induced by additive manufacturing processes, Computer Methods in Applied Mechanics and Engineering, 334, 626-665

2. El-Gizawy, Sherif A., Corl S., Graybill B., 2011, Process-induced Properties of FDM Products, Proceedings of the ICMET, International Conference on Mechanical Engineerings and Technology Congress and Exposition

3. Hoglund R.M., 1992, An anisotropic topology optimization method for carbon fiber-reinforced fused filament fabrication, Ph.D. Thesis, Baylor University, Texas

4. JIANG D., 2017, Three-dimensional topology optimization with orthotropic material orientation design for additive manufacturing structures, Ph.D. Thesis, Baylor University, Texas

5. Kirkpatrick S., Gelatt C.D., Vecchi M.P., 1983, Optimization by simulated annealing, Science, 220, 671-680

6. Lewiński T., Rozvany G.I.N., 2008, Analytical benchmarks for topological optimization IV: square-shaped line support, Structural and Multidisciplinary Optimization, 36, 143-158

7. Liu J., Chen Q., Liang X., To A.C., 2019, Manufacturing cost constrained topology optimization for additive manufacturing, Frontiers Of Mechanical Engineering, 14, 213-221

8. Michell A.G.M., 1904, The limits of economy of material in frame-structures, Philosophical Magazine, 8, 589-597

9. Mirzendehdel A.M., Rankouhi B., Suresh K., 2018, Strength-based topology optimization for anisotropic parts, Additive Manufacturing, 19, 104-113

10. Mirzendehdel A.M., Suresh K., 2016, Support structure constrained topology optimization for additive manufacturing, Computer-Aided Design, 81, 1-13

11. MrzygŁód M., 2012, Multi-constrained topology optimization using constant criterion surface algorithm, Bulletin of the Polish Academy of Sciences - Technical Sciences, 60, 229-236

12. Park J.-H., Goo B., Park K., 2019, Topology optimization and additive manufacturing of customized sports item considering orthotropic anisotropy, International Journal of Precision Engineering and Manufacturing, 20, 1443-1450

13. Querin O.M., Steven G.P., XIE Y.M., 1998, Evolutionary structural optimization (ESO) using bidirectional algorithm, Engineering Computations, 15, 1031-1048

14. Sheth S., Taylor R.M., Adluru H., 2017, Numerical investigation of stiffness properties of FDM parts as a function of raster orientation, [In:] Solid Freeform Fabrication 2017: Proceedings of the 28th Annual International Solid Freeform Fabrication Symposium - An Additive Manufacturing Conference

15. Thore C.-J., Grundstrom H.A., Torstenfelt B., Klarbring A., 2019, Penalty regulation of overhang in topology optimization for additive manufacturing, Structural and Multidisciplinary Optimization, 60, 59-67

16. Xie Y.M., Steven G.P., 1993, A simple evolutionary procedure for structural optimization, Computers and Structures, 49, 885-896

17. Zhang P., LiU J., To A.C., 2017, Role of anisotropic properties on topology optimization of additive manufactured load bearing structures, Scripta Materialia, 135, 148-152

18. Zhou M., Rozvany G.I.N., 1991, The COC algorithm. Part II: Topological, geometrical and generalized shape optimization, Computer Methods in Applied Mechanics and Engineering, 89, 309-336 